\title{
High resolution magnetic resonance techniques for myocardial perfusion and vascular imaging in rodents
}

\author{
Monique Bernard ${ }^{1}$ \\ CRMBM, UMR CNRS 6612, Faculté de Médecine, Université de la Méditerranée, \\ 27 Bd Jean Moulin,13385 Marseille cedex 5, France \\ E-mail: monique.bernard@medecine.univ-mrs.fr \\ Frank Kober \\ CRMBM, UMR CNRS 6612, Faculté de Médecine, Université de la Méditerranée, \\ 27 Bd Jean Moulin,13385 Marseille cedex 5, France \\ E-mail: frank.kober@medecine.univ-mrs.fr

\section{Patrick Cozzone} \\ CRMBM, UMR CNRS 6612, Faculté de Médecine, Université de la Méditerranée, \\ 27 Bd Jean Moulin, 13385 Marseille cedex 5, France \\ E-mail: patrick.cozzone@medecine.univ-mrs.fr
}

\begin{abstract}
As a non invasive and multimodal technique, high resolution magnetic resonance imaging (MRI) allows to measure repetitively in the same animal a variety of physiological and anatomical parameters such as cardiac morphology and function, myocardial blood flow and morphology of the vessels. It is therefore a useful tool to study the pathophysiology of atherosclerotic diseases as well as the therapeutic aspects in animal models. High resolution myocardial perfusion maps can be obtained in vivo in rats and mice using spin labelling techniques to measure non invasively and quantitatively myocardial blood flow in animal models of disease. Microscopic imaging of the vessel wall in small animals can be used as an investigative tool to follow in vivo progression or regression of the atherosclerotic lesion.
\end{abstract}

Frontiers in Imaging Science: High Performance Nuclear Medicine Imagers for Vascular Disease Imaging (Brain and Heart)

Istituto Superiore di Sanita', Rome, Italy

13-14 November, 2006

\footnotetext{
$1 \quad$ Speaker
} 


\section{Introduction}

Animal models hold considerable potential for investigation of the underlying mechanisms of vascular diseases. In particular, genetically engineered mice are being increasingly used to study the influence of genes or environmental factors on the progression of atherosclerosis (APOE-KO mice). Non invasive imaging methods are of particular interest in this area because they allow serial analysis of animal models. Among the existing techniques, magnetic resonance imaging (MRI) has the advantage of providing high spatial resolution and multimodality within a single exam. Mainly two modalities may be of major interest in atherosclerotic diseases. These are quantitative mapping of myocardial perfusion on the one hand, and the ability to perform longitudinal morphological studies of atherosclerotic lesion progression and regression on the other hand.

\section{Myocardial blood flow}

Myocardial perfusion MRI can detect diffuse microvascular alterations associated with impaired endothelial function or localized alterations associated with atherosclerosis. Contrastenhanced first-pass perfusion MRI and arterial spin labeling are two alternative techniques to measure myocardial blood flow. While first-pass MRI using gadolinium contrast agents is easily performed on the human heart, it has a number of disadvantages in small animals, mainly related to high capillary blood flow and high heart rates. Spin labeling MRI is an interesting alternative particularly for animal studies. Spin labeling perfusion quantification is based on the effect of inflowing non inversed spins into the imaging slice in which the magnetization has been inversed. The signal observed with this technique is directly proportional to capillary blood flow making the high flow values encountered in small animals advantageous. Spin labeling perfusion MRI in the mouse heart has been performed using segmented inversionrecovery FLASH [1]. An improved spin labeling gradient echo sequence has been developed by our group [2]. This method provides high sensitivity at high spatial resolutions and the ability to measure myocardial perfusion in freely breathing animals in vivo at high heart rates both in rats [2] and mice [3]. A typical perfusion map obtained with this method in a mouse anaesthetized with isoflurane is shown in figure 1 . Physiological conditions and especially anaesthesia have to be carefully controlled for the determination of this parameter. We have studied the influence of the anaesthesia on myocardial blood flow in both rats [4] and mice [3]. These studies show the importance of the isoflurane concentration due to the vasodilating properties of isoflurane. Microvascular alterations have been demonstrated by measuring myocardial blood flow in rat models of type 2 diabetes [5] and of hypertension superimposed on type 1 diabetes [6]. 


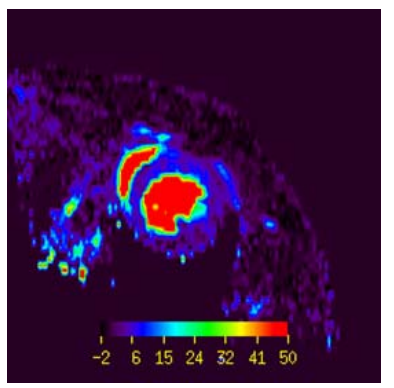

Figure 1: representative color-coded short axis myocardial perfusion (MBF) map obtained at 4.7 $\mathrm{T}$ in a mouse. Anaesthesia was $1.25 \%$ isoflurane.

\section{Vascular imaging in mice by microscopic MRI}

MR microscopy permits to obtain high resolution images of the aorta in mice both at the level of the abdominal aorta [7] and the aortic arch [8]. It is possible to follow progression and regression of the atherosclerotic lesion in the arterial wall [9] and therefore to perform serial analyses. One major difficulty in mouse vessel wall MRI is given by the comparatively small vessel wall thickness. High spatial resolutions are necessary to visualize and to measure the thickness of the arterial wall. A high magnetic field strength is therefore required to maintain sufficient MR sensitivity. Cardiac and respiratory motion and the pulsatile flow in the vessel represent additional difficulties. Measurements in the aortic arch therefore require cardiac and respiratory double-gating although respiratory gating only is sufficient for assessment of the abdominal aorta. Magnetization preparation sequences for blood suppression become necessary depending on the blood flow velocity at the location of interest along the aorta.

The following example illustrates a follow-up vessel wall MRI study in the aorta of $\mathrm{ApoE}^{-/-}$mice at the abdominal level. Precise positioning of the spin-echo MRI slices perpendicular to the abdominal aorta can be obtained using MR angiography images as reference (figure 2). Spin echo MR images then allow to study the time dependent changes in the aortic wall in $\mathrm{ApoE}^{-/-}$mice fed with an atherogenic diet (figure 3). The use of targeting MR contrast agents combined with optimized MRI could add important information about plaque composition.

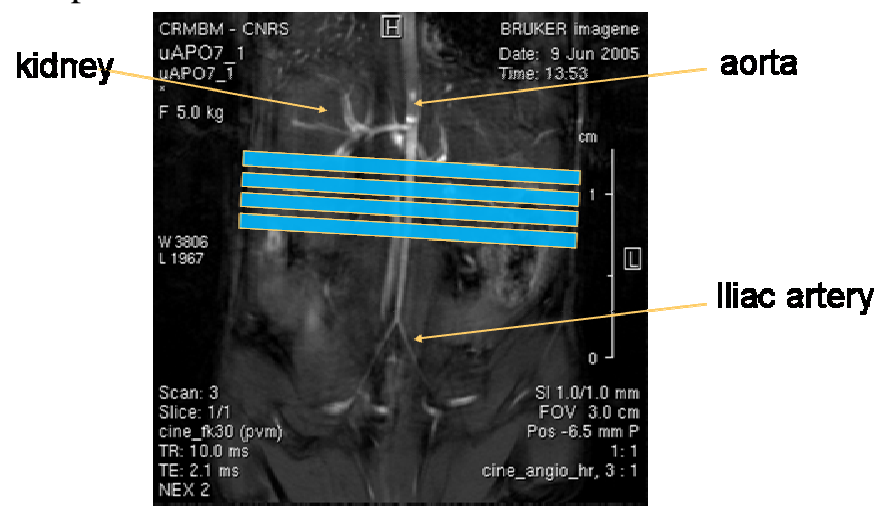

Figure 2: Mouse abdominal MRI at $11.75 \mathrm{~T}$. Localization of image slices using MR angiography as reference. This procedure ensures perpendicular slice positioning with respect to the abdominal aorta. 


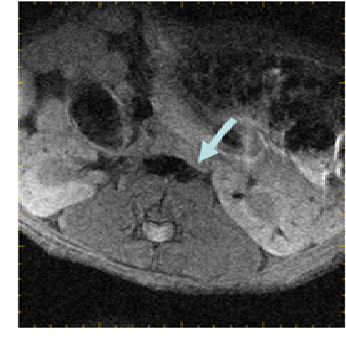

7 weeks

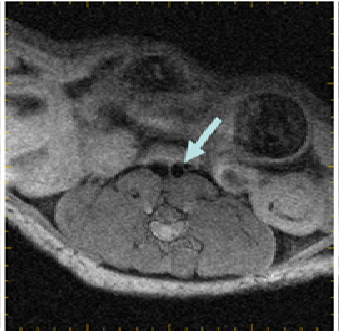

15 weeks

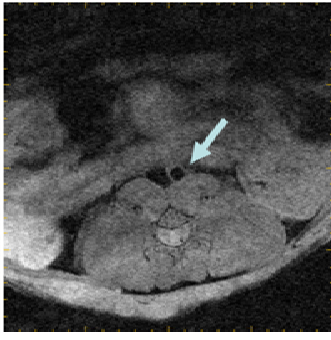

25 weeks

Figure 3: Abdominal aortic wall in ApoE-/- mice fed with an atherogenic diet at different ages. Spin echo MRI, TE=8ms, TR>1500 ms, in-plane resolution: 78x78 mm², slice thickness: $1 \mathrm{~mm}$, acquisition time $=25$ minutes ( 7 slices). Wall thickening over time is clearly visible.

\section{Conclusion}

In the field of vascular diseases, MRI is an interesting tool for its multimodal and high resolution capability. In particular, serial high-resolution studies of myocardial blood flow and plaque morphology in animal models of disease are feasible. These parameters may provide important information on the pathophysiological mechanisms of atherosclerosis. They may also be used in follow-up studies of therapies.

\section{References}

[1] Streif JU, Nahrendorf M, Hiller KJ, Waller C, Wiesman F, Rommel E, Haase A, Bauer WR In vivo assessment of absolute perfusion and intracapillary blood volume in the murine myocardium by spin labeling magnetic resonance imaging. Magn. Reson. Med. 2005; 53: 584-592.

[2] Kober F, Iltis I, Izquierdo M, Ibarrola D, Cozzone PJ, Bernard M. High resolution myocardial perfusion mapping in small animal in vivo by spin labeling gradient- echo imaging. Magn. Reson. Med. 2004; 51: 62-67.

[3] Kober F, Iltis I, Cozzone PJ, Bernard M. Myocardial blood flow mapping in mice using high-resolution spin-labeling MRI : influence of ketamine/xylazine and isoflurane anesthesia. Magn. Reson. Med. 2005; 53: 601-606.

[4] Iltis I, Kober F, Dalmasso C, Lan C, Cozzone PJ, Bernard In vivo assessment of myocardial blood flow in rat heart using MRI : effect of anesthesia. J Magn Reson Imaging. $2005 ; 22$ : 242-247.

[5] Iltis I, Kober F, Desrois M, Lan C, Dalmasso C, Portha B, Cozzone PJ, Bernard M Defective myocardial blood flow and altered function of the left ventricle in type 2 diabetic rats. A non invasive in vivo study using perfusion and cine magnetic resonance imaging. Invest. Radiol. 2005; 40: 19-26.

[6] Iltis I, Kober F, Dalmasso C, Lan C, Cozzone PJ, Bernard M. Non invasive characterization of myocardial blood flow in diabetic hypertensive rats using spin-labeled MRI. Microcirculation. 2005; 12 : 607-614. 
[7] Fayad ZA, Fallon JT, Shinnar M, Wehrli S, Dansky HM, Poon M, Badimon JJ, Charlton SA, Fisher EA, Breslow JL, Fuster V. Non invasive in vivo high-resolution magnetic resonance imaging of atherosclerotic lesions in genetically engineered mice. Circulation 1998; 98 : 1541-1547.

[8] Wiesmann F, Szimtenings M, Frydrychowicz A, Illinger R, Hunecke A, Rommel E, Neubauer S, Haase A. High resolution MRI with cardiac and respiratory gating allows for accurate in vivo atherosclerotic plaque visualization in the murine aortic arch. Magn Reson Med 2003; 50 : 69-74.

[9] Kober F, Canault, M. Mueller C, Peiretti F, Juhan-Vague I, Cozzone PJ, Nalbone G, Bernard M. Longitudinal MRI follow-up of the development of atherosclerosis in mouse aortic arch. Magn Reson Mater Phy 2006; 19 Suppl 7:227-386. 